\title{
Fracture Dislocation of the Proximal Humerus with Diaph- yseal Extension Associated with Wrist Drop in 57 Years Old Gentleman
}

\author{
Mohamed Shaalan, MD* (D), Mohamed Monawar, MRCS and Satish Kutty, MD, FRCS \\ Sligo University Hospital, Ireland

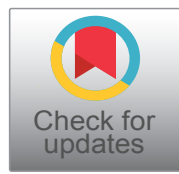

*Corresponding author: Mohamed Shaalan, MD, Sligo University Hospital, Ireland

\begin{abstract}
A 57-years-old gentleman left hand dominant working as a manual labourer in a factory fell from ladder on 16/12/2017, on initial assessment (Full ATLS protocol) there was no injury besides his left upper limb/arm. BP: 139/80 - HR: 70/min - $\mathrm{O}_{2}$ saturation: $97 \%$ on R/A - RR: 18 - Temperature 36.1.

There was swelling and deformity of the left shoulder and arm with intact sensation of the ulnar nerve but diminished sensation of the median and radial nerve with wrist drop and a weak hand grip.

Open reduction of the dislocated shoulder and fracture and plate and screws fixation through combined deltopectoral and lateral approach, The nerve was carefully freed and retracted using vascular loop., postoperative dynamic splint applied, patient discharged home three days post-operative, with regular follow up X-Rays and nerve conduction study. The fracture healed within 4 months and patient had nerve conduction studies performed, which revealed damage at the level of the cords with affection of posterior cord, on second nerve conduction study last May showed evidence of ongoing re-innervations in the upper part of the posterior cord muscles but less so on the lower elements.

Patient is able to manage his daily requirement with his dominant left hand but still off work, ROM on last visit last May, Abduction $0^{\circ}-70^{\circ}$, FF $0^{\circ}-90^{\circ}$, ER $0-45^{\circ}$, IR $0^{\circ}-70^{\circ}$, patient still on his program for physiotherapy.
\end{abstract}

\section{Background}

In complex anterior fracture-dislocations of the proximal humerus, a Neer three- or four part fracture is combined with disruption in the anteroinferior soft-tissue envelope [1], Owing to the devascularisation of the humeral head as a result of the disruption of its capsu- lar attachments, there is a high risk of osteonecrosis or nonunion following this injury [2].

Successful open reduction and internal fixation offers the prospect of an improved functional outcome and the possibility that the development of osteonecrosis may be incomplete or relatively asymptomatic and complete revascularisation of the humeral head may occur through creeping substitution [3].

\section{Case Presentation}

A 57-years-old fit \& healthy gentleman left hand dominant working as a manual labourer in a factory with no known past medical history or allergies.

On the evening of $16 / 12 / 2017$ while attempting to change a light bulb at home he Fell off a ladder \& presented to ED with severe pain left shoulder and arm.

On initial assessment (Full ATLS protocol) there was no injury besides his left upper limb/arm. BP: 139/80 HR: $70 /$ min - $\mathrm{O}_{2}$ saturation: $97 \%$ on R/A - RR: 18 - Temperature 36.1.

On examination of the left upper limb there was no wound. There was swelling and deformity of the left shoulder and arm with intact sensation of the ulnar nerve but diminished sensation of the median and radial nerve with wrist drop and a weak hand grip.

A humeral stabilising system (HSS) brace was applied after obtaining a radiograph of the left humerus which confirmed a fracture dislocation and was admitted to the orthopaedic ward. No attempt to reduce the gleno-

\footnotetext{
Citation: Shaalan M, Monawar M, Kutty S (2020) Fracture Dislocation of the Proximal Humerus with Diaphyseal Extension Associated with Wrist Drop in 57 Years Old Gentleman. Int Arch Orthop Surg 3:024. doi.org/10.23937/2643-4016/1710024

Accepted: December 29, 2020; Published: December 31, 2020

Copyright: (C) 2020 Shaalan M, et al. This is an open-access article distributed under the terms of the Creative Commons Attribution License, which permits unrestricted use, distribution, and reproduction in any medium, provided the original author and source are credited
} 
humeral joint was done in casualty as per local protocol.

The patient was taken to theatre the following day and after general anaesthesia multiple trials of closed reduction of the dislocated shoulder failed. The patient was then positioned supine/beach chair and a combined delto-pectoral (to the proximal) and antero-lateral approach (to the shaft) to the left arm was undertaken. The rotator cuff, long head of biceps and capsule was split and the humeral head reduced easily thereafter, while the greater tuberosity was found to be comminuted, it fell into place once the head relocated.

The fracture site was then carefully exposed as well as the radial nerve which was found to be impaled by the sharp distal end of the proximal fragment. The nerve was carefully freed and retracted using vascular loop. Anatomical reduction was carried out for the two major pieces of the proximal fragments and fixed with a $4.5 \mathrm{~mm}$ cortical screw with compression effect. The main diaphyseal fracture fragments were reduced and fixed with two $4.5 \mathrm{~mm}$ interfragmentary cortical screw with compression effect. We then applied a neutralization plate (LC-DCP 12 holes) with 11 holes filled; the proximal two screws were $6.5 \mathrm{~mm}$ cancellous and the rest $4.5 \mathrm{~mm}$ cortical screws.

Following washout some synthetic bone graft was applied to the fracture site, then the rotator cuff tendons were repaired and a tenodesis of the long head of biceps to the bicipital groove performed.

Soft tissues and skin closed and an opposite dressing applied.

No recovery of radial nerve in the postoperative pe- riod so a dynamic wrist drop splint was applied by our occupation therapy team.

\section{Investigations}

Routine blood test done for him (Table 1).

X-Ray for the Left humerus; fracture dislocation Left shoulder with diaphyseal extension (Figure 1, Figure 2, Figure 3, Figure 4 and Figure 5).

\section{Outcome}

The fracture healed within 4 months and patient had nerve conduction studies performed on two occasions the last on 11.05.2018 (posterior cord/triceps/supinator/EDC/ECR/EIP screening) which was reported as: Positive sharp waves with polyphasic recruitment for the triceps in the order of $60 \%$. The supinator showed positive sharp waves, fibrillation potential with recruitment pattern at $40 \%$ normal which is variable and showing ongoing re-innervation. EDC/ECR/EIP showed positive sharp waves but no particular motor units noted with evidence of ongoing re-innervation in the upper part of the posterior cord muscles but less so on the lower elements. Still no active fingers or wrist extension.

Patient is able to manage his daily requirement with his dominant left hand but still off work, ROM on last visit last May, Abduction $0^{\circ}-70^{\circ}$, FF $0^{\circ}-90^{\circ}$, ER $0-45^{\circ}, \mathrm{IR}$ $0^{\circ}-70^{\circ}$, patient still on his program for physiotherapy.

On last visit on $10^{\text {th }}$ August there is a recovery of the wrist extensors grade IV and to some extent of the finger extensors Grade III, recovery of sensation of the hand.

Table 1: Routine blood test done for him.

\begin{tabular}{|l|l|l|l|l|l|l|l|l|l|l|l|l|l|}
\hline Dates & CRP & Platelets & Hb & WBCS & Neutrophils & INR & 137 \\
\hline $16 / 12 / 2014$ & 1 & 262 & 13.5 & 16.5 & 15.18 & 1.4 \\
\hline
\end{tabular}

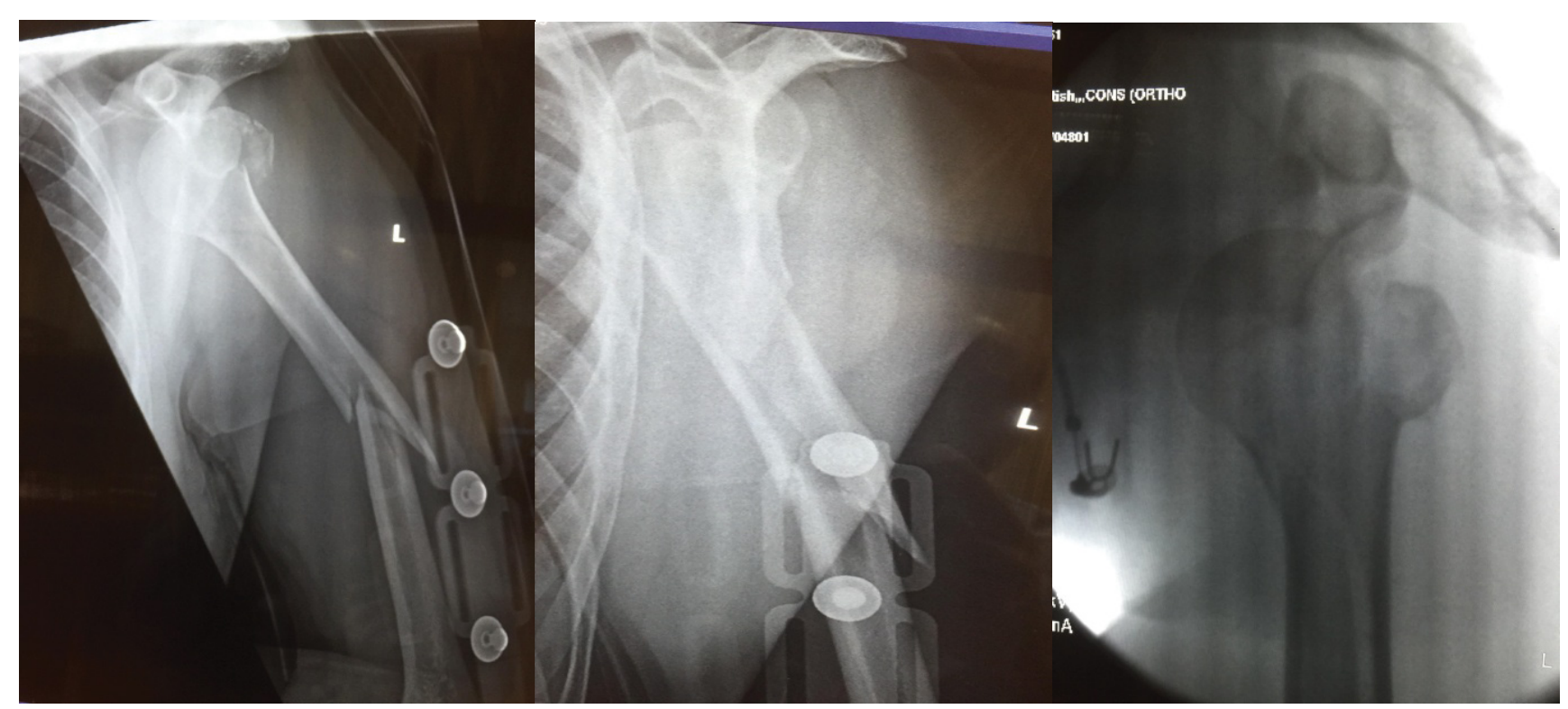

Figure 1: Preoperative X-ray. 

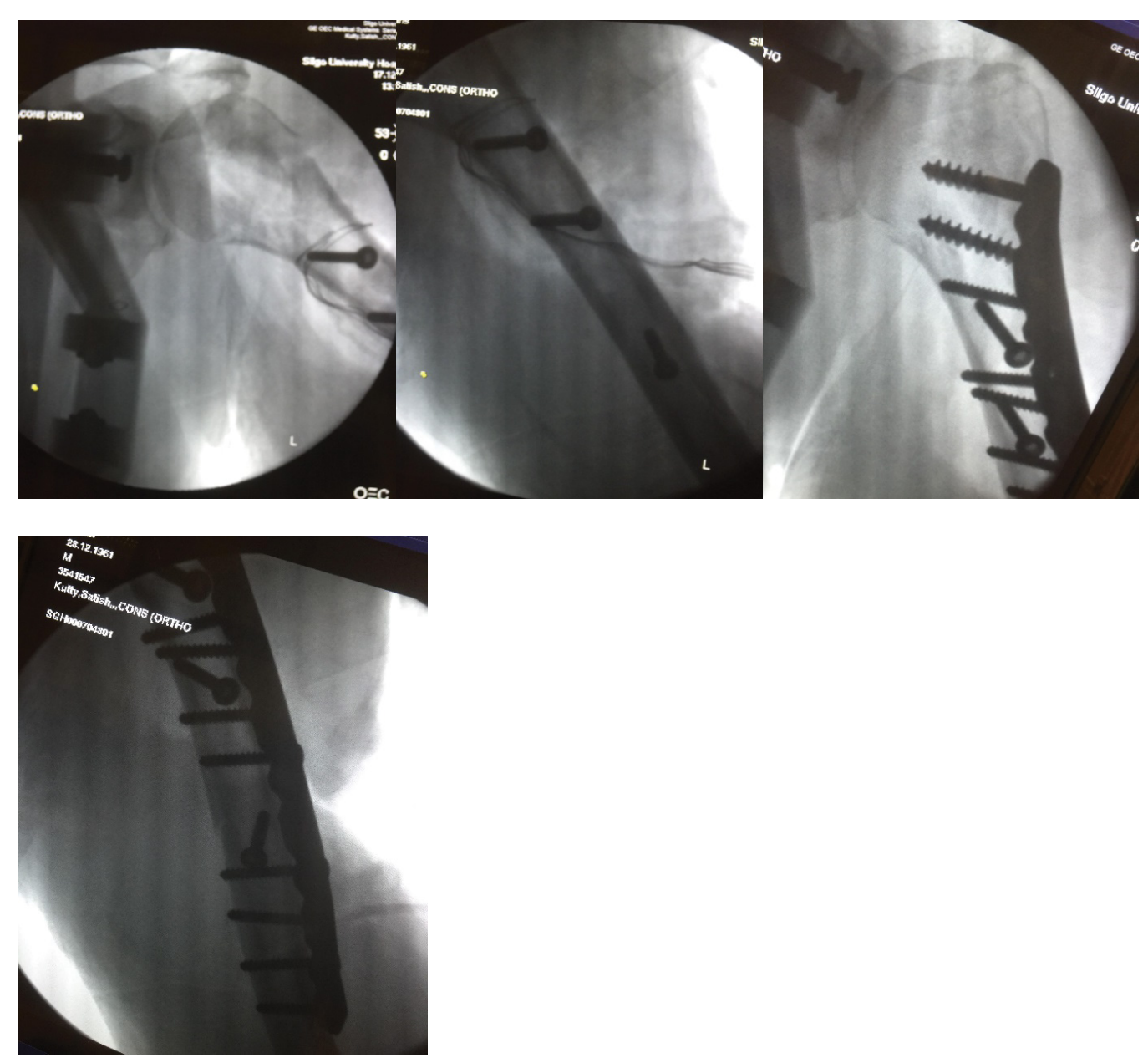

Figure 2: Intraoperative images.

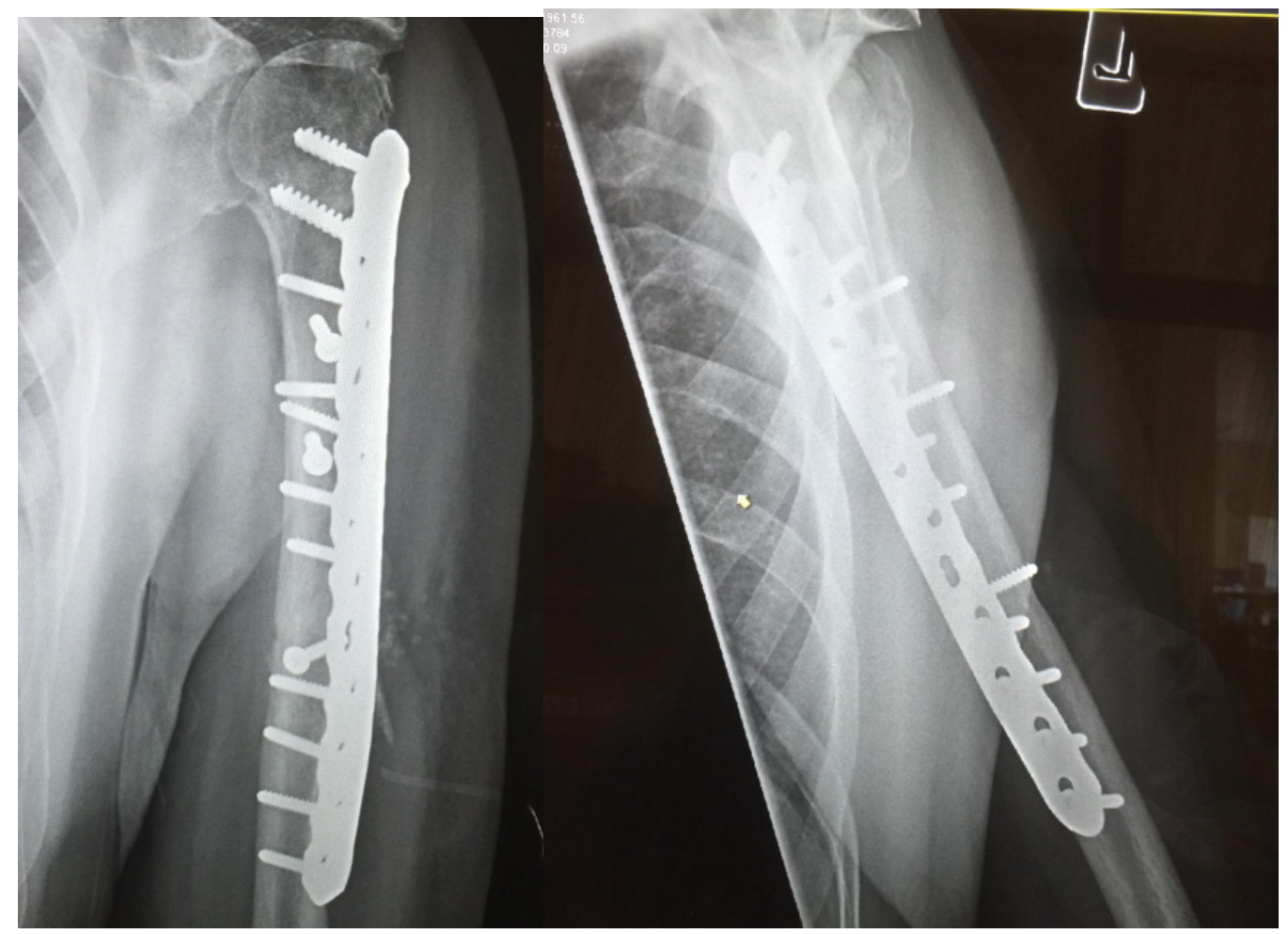

Figure 3: F/U X-ray 3/12. 

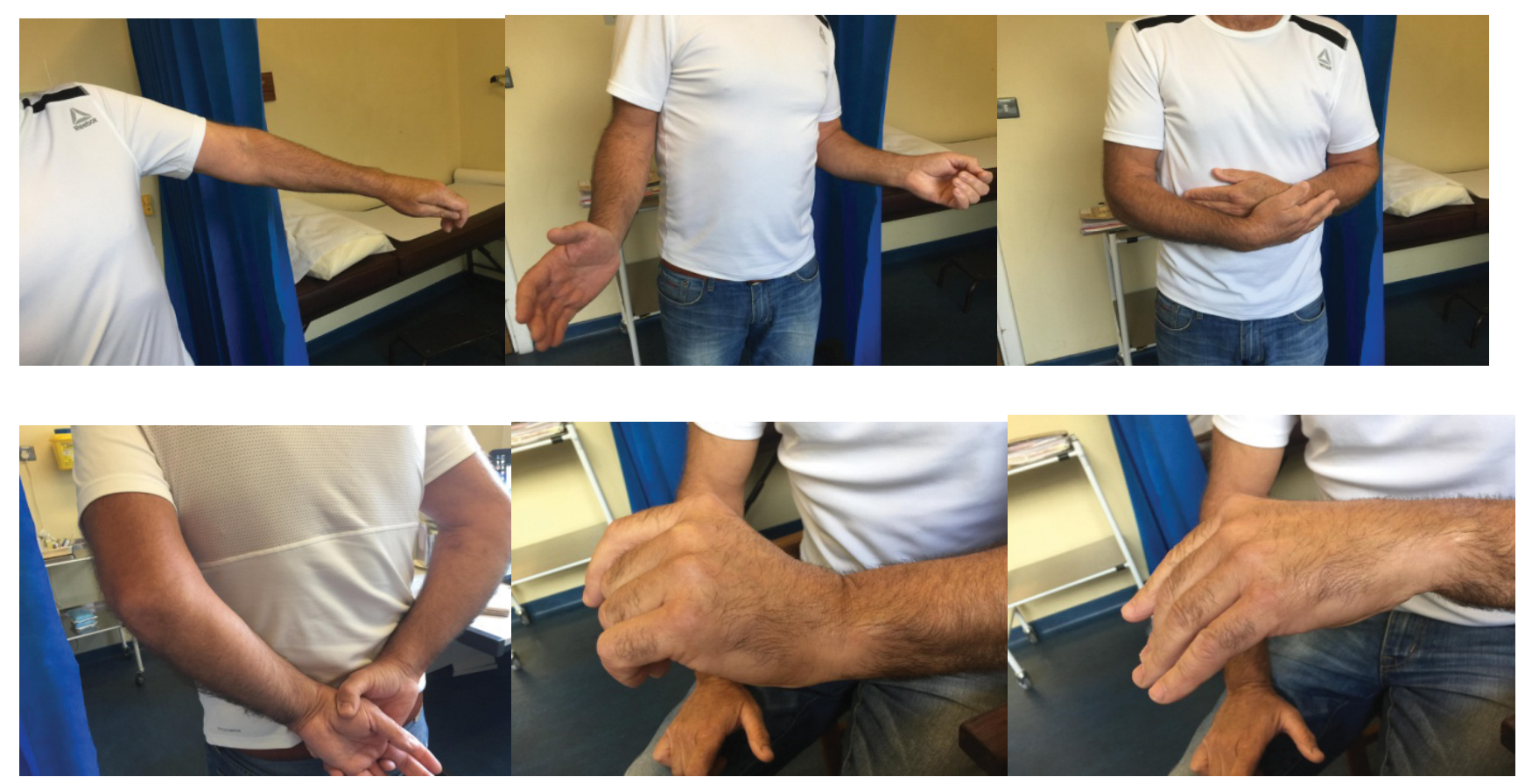

Figure 4: Clinical recovery of brachial plexus injury.

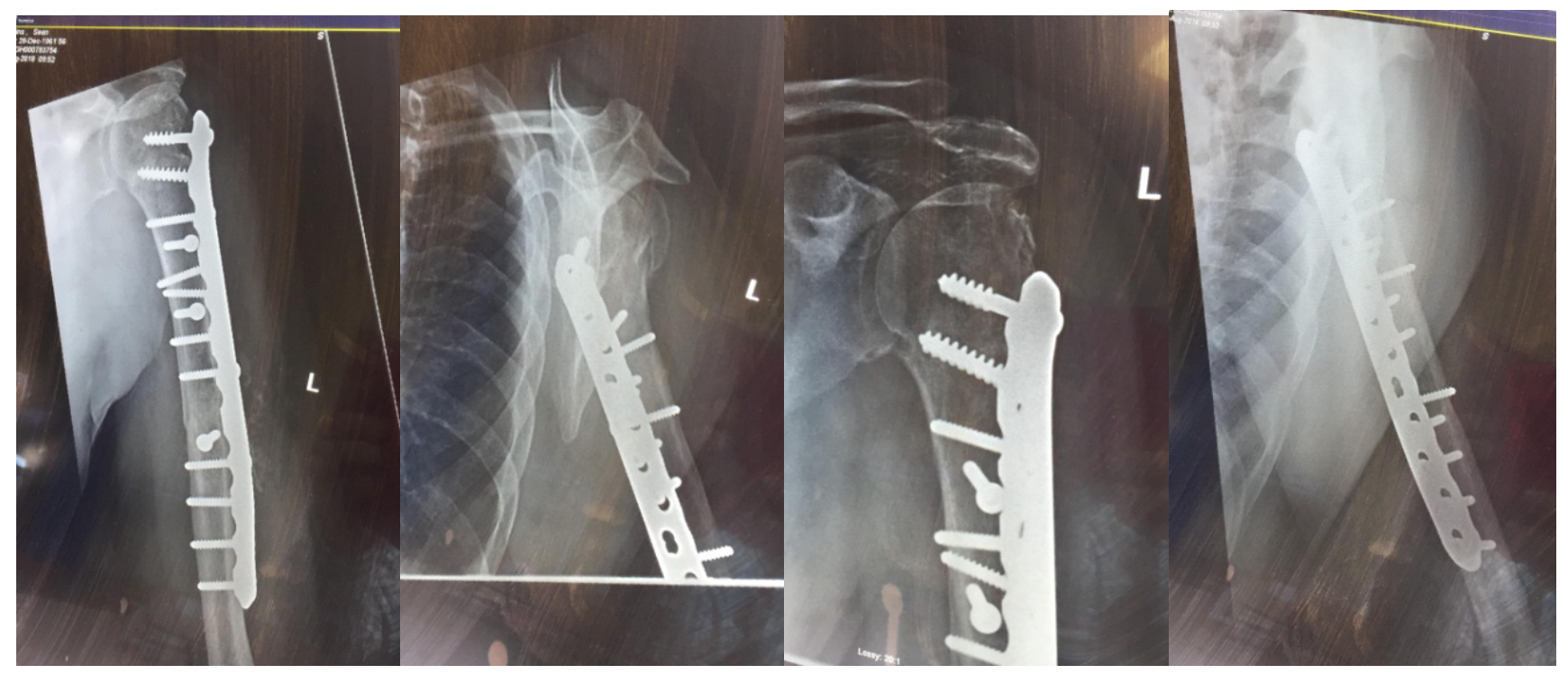

Figure 5: F/u X-ray 6/12.

\section{Discussion}

To our knowledge and literature review three parts fracture dislocation of the proximal humerus with ipsilateral diaphyseal extension is quite seldom seen, the point also the approach for shoulder reduction, exploration of radial nerve and fixation with long plate to address the fracture, this is the reason of combination of deltopectoral proximally and lateral approach distally, best management is open reduction and internal fixation but some of the authors tried conservative treatment, Karimi-Nasab reported management of fracture and dislocation conservatively [4]. Flint reported the conservatively managed for the case of dislocation with three parts proximal humerus fracture with shaft of humerus fracture [5].

\section{Conclusion}

Fracture dislocation of the humerus is rare and a challenging task. It is not possible to treat this patient conservatively, results of open reduction of internal fixation unexpected especially with posterior cord concussion with wrist and finger drop, still the risk of avascular necrosis high but not obvious early, may manifest later, stiffness is the most common sequel of this kind of trauma and fracture.

\section{References}

1. Court-Brown CM, Garg A, McQueen MM (2001) The epidemiology of proximal humeral fractures. Acta Orthop Scand 72: 365-371.

2. Gerber C, Hersche O, Berberat C (1998) The clinical rele- 
vance of posttraumatic avascular necrosis of the humeral head. J Shoulder Elbow Surg 7: 586-590.

3. Kofoed H (1983) Revascularisation of the humeral head: $A$ report of two cases of fracture-dislocation of the shoulder. Clin Orthop Relat Res, 175-178.

4. Karimi-Nasab MH, Shayesteh-Azar M, Sajjadi-SaraviM,
Daneshpoor SMM (2012) Anterior shoulder dislocation and ipsilateral humeral shaft fracture. Iran J Med Sci 37: 202204.

5. Flint JH, Carlyle LM, Christiansen CC, Nepola JV (2009) Case report and literature review anterior shoulder dislocation with three-part proximal humerus fracture and humeral shaft fracture. lowa Orthop J 29: 105-113. 